\title{
A planar bilayer lipid membrane biosensor based on enzymes in the service of environmental monitoring
}

\author{
K. N. Georgopoulos \& C. G. Siontorou \\ Laboratory of Simulation of Industrial Processes, \\ Department of Industrial Management and Technology, \\ School of Maritime and Industry, University of Piraeus, Greece
}

\begin{abstract}
The purpose of this study is the design of a biosensor taking into account the needs, the existing parameters and the environmental constraints in various ecosystems. Real time measurements from such biosensor could lead to reliable decisions about the status of an ecosystem. Two different approaches are presented in this paper. The first is a knowledge-based approach aimed to create an information tool suitable for measuring the critical parameters of a specific ecosystem. The second is an analytical development approach of the construction of a horseradish biosensor for peroxide detection.

Keywords: environmental monitoring, water pollution, lipid bilayer, membranes, enzyme, environmental biosensor, hydrocarbons, horseradish biosensor, peroxide detection, transmembrane ion, enzyme immobilization.
\end{abstract}

\section{Introduction}

People living and working in rural areas are at risk of exposure to a wide range of pollutants emitted from agricultural operations. Exposure to pesticides and herbicides is a significant concern. Roughly only $85 \%$ of sprayed airborne pesticides reach their target destination; the remaining $15 \%$ is distributed elsewhere as pesticide drift. Thereby, in situ monitoring of these substances in rivers or lakes is essential nowadays. Enzyme-based biosensing offers a suitable detection means for fast tracking a variety of harmful substances. Selectivity, specificity, response times and detection limits can be easily controlled towards individual compounds or groups of compounds on customized engineered lipid 
membrane platforms to suit any monitoring needs. This paper presents a methodological framework for the design and development of a computer-aided enzyme biosensor system. The scope is to provide the validation parameters that will ensure a successful system delivery for environmental monitoring. The proposed methodology has been implemented in the design of an enzyme biosensor for the qualitative, quantitative, real time and in situ detectionmonitoring of pollutants in rivers or lake water. For the design of that (BLM) enzyme biosensor, two approaches were followed. The first is a knowledgebased approach for the detection of polycyclic aromatic hydrocarbons (PAHs) in estuaries to identify the constraints, needs and parameters of the operating environment. The second approach is the biosensor manufacture in laboratory scale with the aid of immobilized horseradish peroxidase (HRP) on selfassembling lipid membranes of phosphatidylcholine. Although the shelf life, workability, robustness and some other parameters require further study for the commercialization of the device, it became apparent that, this BLM enzyme biosensor system is suitable for monitoring environmental contaminants.

\section{Methodological framework}

The methodological framework designed for the development of a biosensor custom for the environmental needs is shown in fig. 1. In this framework a knowledge-based approach is used to create an information tool suitable for measuring the critical parameters of a specific ecosystem. Therefore, the opportunity is given for an immediate decision on the state of the ecosystem. A huge number of different kinds of organisms share an ecosystem. Usually, one or more populations are better adapted to the conditions of the system becoming dominant. All other populations usually present as minorities within the same system. The degradation mechanism of the dominant team's cells is usually more selective and more sensitive to pollutant management than other minority populations in the system. Therefore, dominant species could be used as biorecognition elements, since they could be detected easily (step 5). Thus, these cells could be considered as a pollution impact simulator, playing both roles simultaneously, an active role in the degradation of the toxicant and a passive role of the impact recipient. The simulator had to be analysed in its functional subsystems so that the cellular mechanisms that participate more in pollution management are clarified (step 7). Thereby, biosensor systems can be developed for the monitoring of the ecosystem status (step 9). The proposed system could be used for bio monitoring or bio remediation projects, such as the identification of representative species of an ecosystem, the relation of the damage to systemic changes, the optimization of the biotic parameters and the validation of genetically engineered species. 
1. Characterization of ecosystem

a. Specific biochemical and climatic characteristics

b. Relevant pollution sources and pollution history

c. Identification of host factors interrelating exposure to biological effects

\section{$\forall$}

2. Definition of objectives

a. Determination of the pollutants - target analyte

b. Identification of the predominant or consortium species

c. Determination of scope (monitoring, assessment, toxicity)

3. Species ontology relevant degradation pathways

a. Data gathering on genera metabolism

b. Pathways correlation to target analyte

c. Reforming as ontological network
4. Ex-situ elucidation/verification of antistress mechanism

a. Site sampling and isolation of the predominant species

b. Development of microbial sensor for correlation metabolic changes

5. Selection of the target - specific species

a. Multi criteria ranking of the microbial sensors as per sensitivity, selectivity, detection limits, specificity, interference, response time

b. Sensitivity analysis

c. Characterization of species in the first ranged sensor (stability, nutrient dependency growth, community behavioral, cell-cell communication)

6. Decomposition of the system

a. Decomposition of the cellular mechanism into enzymatic sub system

b. Classification of the sub-system into primary and secondary

7. Re-composition of the system

a. Remodeling of the system to include the direct subsystems

b. Identification of signal amplification mechanisms
8. Design of enzyme relevant biosensors

a. Data gathering on biosensors developed on these enzymes

b. Multi-criteria ranking of the enzyme sensors as per sensitivity, selectivity, detection limits, sensitivity

c. Sensitivity analysis

9. Re-composition of the system

a. Remodeling of the system to include the direct subsystems

b. Identification of signal amplification mechanisms

10. In-situ calibration

a. Development for site implementation

b. Feedback and reload to stage 4

Figure 1: The methodological framework designed to support biosensor development custom-tailored to environmental needs. 


\section{A knowledge-based approach}

The methodological framework described has been implemented in the development of the environmental biosensor for the detection of polycyclic aromatic hydrocarbons (PAHs) in estuarine areas. These hydrocarbons are usually found in sediments buried and persist there till their degradation, resuspension, bioaccumulation and removal by river sludge deposition (Filonov et al. [1]). Nowadays, much is known about the rate and chemical pathways of PAHs in water or sediment interfaces. Toxicological data for some of the lowermolecular-weight aromatic hydrocarbons, such as naphthalene, puts them on top of the priority concern list. There are several different factors that affect the degradation of naphthalene within an ecosystem [2, 3]. Alongside the degradation function, microorganisms are involved, such as fungi or bacteria acting independently (step 1c). The acting communities have been limited down to four consortia (step 2b), in which $\gamma$-Proteobacteria can be considered as the predominant consortium in the ecosystem under investigation. This species is commonly used in biosensors (Paton et al. [4]). After constructing the ontological network of the possible metabolic pathways (step 3c), thinning yielded the deoxygenize-based breakdown process (Merks and Glazier [5]) as the most possible mechanism under the conditions studied (step 7a). The biodegradation of naphthalene has several stages which are performed mainly by oxygenates and consumption of oxygen resulting in the breakdown of the aromatic rings. Naphthalene deoxygenase activates cell respiration, justifying the suitability of the species for microbial sensor development. All the reactions shown involve electron transfer, thus enabling the utilization of the enzyme subsystems in amperometric biosensing. Six such sub-systems have been identified as suitable for step 9a, relating to naphthalene detection, pathway verification and metabolite screening. As it was found out, the presence of naphthalene inhibits the reaction, making the development of an indirect biosensor possible, where the decrease of pyruvate concentration could be related to naphthalene concentration in samples (Van Hamme et al. [3]). The subsequent reaction involving salicylaldehyde dehydrogenase serves in vivo as a signal amplifier (step 7b) and should be included in the biosensor format. Pathway selection and/or predominance have been correlated to plasmid regulators and assumed to be controlled genetically as a unit. However, recent studies suggest that although the pathways may be readily interchanged in vivo, their regulation is not genetically controlled, making plasmid extrapolation for molecular engineering largely ineffective. The clarification of the pathway followed and the study of the conditions under which each path is activated, is vital to ecotoxicology engineering. The proposed scheme, exploiting site-derived anti-stress response mechanisms as a molecular tool for developing site-specific field measuring systems, integrates effectively multiple layers of knowledge in order to link abiotic parameters to ecological quality and provide the necessary information granularity in a single assessment framework. Thereby, the developed biosensors assess the ecological effects of pollution rather than pollution itself, guiding advances on ecological microbiology, bioremediation and biomonitoring. 


\section{Construction of horseradish (HRP) biosensor for peroxide detection: analytical development}

Understanding interactions between proteins and surfaces is very important in many fields of biomedical science, from biosensors to biocompatible materials. Peroxidases, in general, catalyse oxidation of a large variety of substrates by hydrogen peroxide. This reaction gives an efficient way for removal of toxic hydrogen peroxide from the intracellular region (Chattopadhyay and Mazumdar [6]). It is now accepted that peroxidases occur as a large family of isoenzymes. Isoenzymes are different molecular forms of the same enzyme, which catalyse the same biochemical reaction, but have distinct physical, chemical and kinetic properties arising from small differences in their amino acid sequence (Veitch [7]). Applications of HRP in analytical detection of peroxidases have considerable potential for application in many different areas. Nevertheless, their commercial application is especially well established in analytical diagnostics, especially in biosensors and immunodetection. Moreover, the nature of the catalysed reaction also allows the use of electrochemical detection procedures, and thus the development of amperometric biosensors. As an example of the implementation of stages 8 and 9 of the methodological framework, this work involves the construction of an HRP amperometric biosensor for the detection of commercial-grade $\mathrm{H}_{2} \mathrm{O}_{2}$. Since HRP is capable of reducing $\mathrm{H}_{2} \mathrm{O}_{2}$ and also some organic peroxides, HRP-based biosensors are widely used to detect these peroxides for environmental biomonitoring. Several HRP based biosensors for the detection of small molecules other than $\mathrm{H}_{2} \mathrm{O}_{2}$ have been developed in recent years, which include peroxides as biological detection element (Zhang and $\mathrm{Li}$ [8]). The HRP-based biosensors are also the most sensitive to a great number of compounds that act as electron donors, such as phenolic compounds. In bio electrochemistry, peroxidase studies have been numerous, and since the late eighties considerable interest has been paid to the development and characterization of HRP-modified electrodes [9-11]. The mechanism for the compound detection is based on the direct electron transfer between the enzyme and the electrode matrix, allowing the construction of electrochemical biosensors [12]. HRP is capable of reducing $\mathrm{H}_{2} \mathrm{O}_{2}$ and also some organic peroxides, in a rather simple procedure and the principle of detection is described by two chemical equations (eqn (1) and eqn (2)).

$$
\begin{gathered}
\mathrm{HRP}_{\text {ferric }}+\mathrm{H}_{2} \mathrm{O}_{2} \rightarrow \mathrm{HRP}_{\text {compound } 1}+2 \mathrm{H}_{2} \mathrm{O} \\
H R P_{\text {compoundl }}+2 e^{-}+2 \mathrm{H}^{+} \rightarrow \mathrm{HRP}_{\text {ferric }}+\mathrm{H}_{2} \mathrm{O}
\end{gathered}
$$

If an HRP-modified electrode is placed in a solution containing a peroxide $(\mathrm{ROOH})$ and set at a sufficiently negative potential, then a proportionality between the reduction current and the peroxide concentration is observed (the peroxide oxidizes the enzyme and the electrode reduces it back to its native form) (Azevedo et al. [13]). Mediators, small redox molecules (e.g. ferrocene$\mathrm{Fc}$ ), can be used to enhance the intensity of the current generated. 


\section{Materials and methods}

\subsection{Transmembrane ion transport in BLMs: experimental aspects}

To transport solute or solvent molecules across a membrane, such as a BLM, a driving force must be present, i.e. a gradient of chemical potential, electrochemical potential, electrical potential, pressure, or temperature. The pathway across the BLM may be facilitated by carriers or channels. Experimental evidence indicates that the resistance to solute permeation is most likely located in the interfacial region, although the membrane phase (lipid bilayer core) can also be the rate-limiting barrier. As expected, the rate of material transport, across BLMs increases with increasing temperature, being particularly so at the phase transition temperature. However, other factors such as ion-binding and lipid flip-flop rate at increased temperature may be also important. Movements of charged species (ions, electrons, holes) across a BLM constitute electric currents. Electrical parameters that have been measured on BLMs are membrane potential $(\mathrm{Em})$, resistance $(\mathrm{Rm})$, current $(\mathrm{Im})$, capacitance $(\mathrm{Cm})$, breakdown voltage $(\mathrm{Vb})$ and $\mathrm{I} / \mathrm{V}$ curves (voltammograms) (Ottova and Tien [14]).

\subsection{Materials}

The materials used throughout this study were:

- Lipid L- $\alpha$-phosphatidylcholine from egg yolk (Type XVI-E 99\% TLC);

- Silver wire with a diameter of $0.1 \mathrm{~mm}$;

- Enzyme Peroxidase, type XII from horseradish (1.7 mg solid);

- Peroxide solution 3\% w/w (commercial grade);

- Water, distilled through a filtering system.

\subsection{Biosensor set-up preparation of s-BLMs}

A two-electrode configuration was used throughout the experiments and consisted of a sensing electrode (silver wire with an s-BLM) and an Ag/AgCI electrode acting as a reference. An external d.c. potential of $25 \mathrm{mV}$, provided by a power source (model: 2400, SourceMeter ${ }^{\circledR}$ KEITHLEY) was applied between the electrodes and the ionic current through the BLM was measured with a digital electrometer (model: 6514, System Electrometer KEITHLEY). The same electrometer was used as a current-to-voltage converter. The electrochemical cell and sensitive electronic equipment were placed in a grounded Faraday cage. The lipid solution used for the formation of the metal supported BLMs contained $2.5 \mathrm{mg} / \mathrm{ml}$ of lipid. The freshly cut tip of the electrode was dipped into the lipid solution and subsequently immersed into a $0.1 \mathrm{M}$ KCI aqueous solution. The lipids were self-organized into a micelle-type formation around the electrode tip, in such a way that the polar heads of the lipids were tangent to the metal surface and the tails were placed inside the micelle. The micelle starts to collapse due to the electrostatic interactions, directing the lipid molecules towards the metal 
attached monolayer. After 10-40 minutes, any multi-layer formation was broken down, leaving a clear bilayer insulating the tip of the electrode immersed in the water. The electrochemical current was stabilized over a period of about $22 \pm 13 \mathrm{~min}$. All experiments were performed at $20-25 \pm 1^{\circ} \mathrm{C}$.

\subsection{Enzyme immobilization}

The selectivity of the BLMs towards hydrogen peroxide was obtained by introducing exact concentrations in each experimental procedure of stock horseradish peroxidase solution into the aqueous electrolyte solution, ranging through a decade of concentrations $(0.17-1.7 \mu \mathrm{g} / \mathrm{ml})$. When the ion current stabilized (over a period of a. $20 \mathrm{~min}$ ) and no more transient current changes occurred (other than the noise peaks), hydrogen peroxide was injected into the bulk electrolyte solution. In order to investigate the degree of in solubilisation of the enzyme into the bilayer structure, and possibly to optimize enzyme loading, the lipid forming solution was enriched with $0.34 \mu \mathrm{g} / \mathrm{ml}$ of the enzyme, allowing for both bilayer formation and enzyme incorporation to proceed in a single step. After the preparation of the mixture, the metal wire, which was freshly cut, was immersed in the mixture. The wire coated with both the lipid solution and the enzyme was subsequently immersed into an aqueous solution $0.1 \mathrm{M} \mathrm{KCI}$. When the ion current stabilized (over a period of $25 \pm 5 \mathrm{~min}$ ) and no more transient current changes occurred (other than the noise peaks), hydrogen peroxide was injected into the bulk electrolyte solution.

\section{Results}

\subsection{Stabilisation of BLM}

The mean stabilization time for BLM formation is $22 \pm 13 \mathrm{~min}$, whereas the background current leveled at $120 \pm 50 \mathrm{pA}$ and the noise level (estimate) was $33 \pm 25 \mathrm{pA}$. The breakdown voltage, i.e., the point after which the relation between ion current and voltage becomes non-linear, has been estimated at $105 \mathrm{mV}$, which is in accordance with values reported for metal supported lipid films from lipid solutions ranging from 100-500 mV (Tien et al. [15]). The estimated value, however, is lower than the values reported in the literature for freely supported BLMs (around $350 \mathrm{mV}$ ), and even lower than the values given for metal supported BLMs produced in situ by lipid vesicles (around $500 \mathrm{mV}$, for this type of lipid) (Dimitrov and Jain [16]). Membrane regeneration in situ, i.e., from the lipid solution already present into the electrolyte cell, was not possible, mainly due to the high cell volume $(57 \mathrm{ml})$ used and the consequent high dispersion of the lipid molecules. Re-forming of membrane was only possible by redipping the electrode (after tip-cutting) into the original lipid forming solution. Once formed and stabilised, the operational stability of the membrane was satisfactory ( $95 \%$ of the times observed), even at high ambient temperatures $\left(32 \pm 2{ }^{\circ} \mathrm{C}\right)$, although in the latter case, a substantial increase of the noise level was observed. The membrane was stable for over 8 hours (within 
the experimental runs), showing a drift of 5\% in the background ion current after 9 hours of continuous operation.

\subsection{Immobilization of enzyme-Incorporation of enzyme}

The enzyme ( $5 \mu \mathrm{l}$ of the stock solution giving a final concentration of $1.7 \mu \mathrm{g} / \mathrm{ml})$ was injected close to the electrode tip. The injection of the enzyme produced high current transients, indicative of the interaction of the enzyme with the lipid bilayer, that were gradually reduced after ca.20 min. The average background current was similar to that of a BLM alone, indicating no pore formation of the lipid bilayer. However, the lower noise level may indicate a less fluid lipid structure, possibly due to surface interaction of the HRP with the membrane. These interactions are not due to electrostatic forces between the lipids and the HRP, but are rather due to enzyme aggregation at the bilayer surface. The stabilization time was the same at lower concentrations of the enzyme, giving comparable background ion currents. The use of a lower enzyme concentration $(0.17 \mu \mathrm{g} / \mathrm{ml})$ gave significantly increased noise levels (ca. $50 \pm 55 \mathrm{pA})$, that prohibited any further experimentation. The incorporation of enzyme into a forming bilayer gave similar results, with ion current stabilization times of $25 \pm$ 5 min. The results indicate that HRP does not span the membrane, supporting the aggregation mechanism.

\subsection{Detection of peroxide}

The introduction of peroxide in the bulk electrolyte solution resulted in permanent ion current increases, the magnitude of which depended on the concentration of the analyte (as cumulative concentration levels). The response of the sensor to the peroxide concentration of $0.05-1.58 \mathrm{mg} / \mathrm{ml}$, is provided as calibration curve. The analyte was injected as constant $\mu \mathrm{l}$ volume additions (after the appearance of the signal and the stabilization of the system). In any case, the increase of the electrolyte volume in the cell was less than $1 \%$. The enzyme biosensor offers 20 times more sensitive response than the membrane biosensor alone. Optimization of analytical signal of the BLM-based sensor toward peroxide in $0.1 \mathrm{M} \mathrm{KCl}$ electrolyte solution may be achieved by the use of different enzyme concentrations (e.g. $0.34 \mu \mathrm{g} / \mathrm{ml}$ ). Concentrations of HPR less than $0.34 \mu \mathrm{g} / \mathrm{ml}$ did not provide adequate sensitivity for detection of peroxide. The use of lower concentration of the enzyme $(0.34 \mu \mathrm{g} / \mathrm{ml})$ produced a similar response profile, with permanent ion current increases, the magnitude of which is dependent on the concentration of the analyte in the bulk electrolyte solution. However, the sensitivity of the sensor toward the analyte was much reduced for the high concentration range, but slightly increased for the low concentration range: the noise level (i.e., $25.95 \mathrm{pA}$ ) sets the detection limit of the sensor (calculated as three times the noise level) at $0.05 \mathrm{mg} / \mathrm{ml}$. The use of lipid films incorporating the enzyme during self-assembly (at enzyme concentration of $0.34 \mu \mathrm{g} / \mathrm{ml}$ ) produced a similar response profile, with permanent ion current increases, the magnitude of which is dependent on the concentration of the analyte in the bulk electrolyte solution. However, the sensitivity of the sensor 
toward the analyte (for the same enzyme concentration) was slightly increased for the high concentration range, but slightly decreased for the low concentration range: the high noise level (i.e., $43.5 \mathrm{pA}$ ) sets the detection limit of the sensor (calculated as three times the noise level) at $0.28 \mathrm{mg} / \mathrm{ml}$.

\section{Commentary on results}

This work describes a technique for the rapid and sensitive electrochemical flow injection monitoring and analysis of peroxide using stabilized systems of supported bilayer lipid membranes (s-BLMs) composed of egg phosphatidylcholine (egg PC) with incorporated HRP. Membrane regeneration in situ was not possible, mainly due to the high cell volume. Re-forming of the membrane was only possible by re-dipping the electrode (after tipcutting) into the original lipid forming solution. Once formed and stabilised, the operational stability of the membrane was satisfactory ( $95 \%$ of the times observed). The membrane was stable for over 8 hours (within the experimental runs), showing a drift of $5 \%$ in the background ion current after 9 hours of continuous operation. The bilayer shows a small response to peroxide, possibly due to the accumulation of the charged molecules on the bilayer surface that induce a slight increase in the surface charge density of the membrane. The response time, on the order of $1 \pm 2 \mathrm{~s}$, supports this view, indicating rapid alterations at the membrane surface. We also examined the physicochemical interaction between the enzyme (HRP) and the membrane. The injection of the enzyme $(1.7 \mu \mathrm{g} / \mathrm{ml})$ produced high current transients, indicative of the interaction of the enzyme with the lipid bilayer, that were gradually reduced after ca. 20 min. The average background current was similar to that of a BLM alone, indicating no pore formation of the lipid bilayer. However, the lower noise level (16.86 \pm $3.934 \mathrm{pA}$ ), may indicate a less fluid lipid structure, possibly due to surface interaction of the HRP with the membrane (since the egg-PC used in this study is not strongly polar whereas HRP is) these interactions are not due to electrostatic forces between the lipids and the HRP, but rather due to enzyme aggregation at the bilayer surface. The stabilization time was the same at lower concentrations of the enzyme $(0.34 \mathrm{mg} / \mathrm{ml})$, giving comparable background ion currents. It can be noticed, however, a slight increase of the average noise level, as well as a higher variability between the individual values. It seems that the lower the concentration of the enzyme the less the aggregation at the membrane surface, but still there is no evidence of membrane spanning or other hydrophobic interaction with the membrane (as monolayer perturbation or flip-flop). It was also concluded that any signal observed was due to electrostatic and/or thermodynamic alterations of the lipid bilayer since reproducible and relatively high ion currents were achieved by introducing the enzyme into the electrolyte solution after the lipid film thinned to a bilayer structure. The introduction of peroxide in the bulk electrolyte solution resulted in permanent ion current increases, the magnitude of which depended on the concentration of the analyte (as cumulative concentration levels). The response times (to establish $99 \%$ of steady-state current) of a sensor incorporating $1.17 \mu \mathrm{g} / \mathrm{ml}$ of HRP was $<1 \mathrm{~s}$. The 
enzyme biosensor offers 20 times more sensitive response than the membrane biosensor alone. The use of lower concentration of the enzyme $(0.34 \mu \mathrm{g} / \mathrm{ml})$ produced a similar response profile, with permanent ion current increases, the magnitude of which is dependent on the concentration of the analyte in the bulk electrolyte solution. However, the sensitivity of the sensor toward the analyte was much reduced for the high concentration range, but slightly increased for the low concentration range: the noise level (i.e. $25.95 \mathrm{pA}$ ) sets the detection limit of the sensor (calculated as three times the noise level) at $0.05 \mathrm{mg} / \mathrm{ml}$. Another alternative technique of enzyme incorporation from which several conclusions have been drawn indirectly is the incorporation of enzyme into a forming bilayer gave similar results, with ion current stabilization times of $25 \pm 5 \mathrm{~min}$. The results indicate that HRP does not span the membrane, supporting the aggregation mechanism. Moreover, the higher residual currents (compared to those obtained when the enzyme incorporated into a pre-formed lipid film) of $240 \pm 54 \mathrm{pA}$ (with a noise level of $43.5 \mathrm{pA}$ ) suggest limited enzyme diffusion into the bulk electrolyte, making more enzymes available for surface aggregation. Thus, this technique enhances the capacity of the bilayer for enzyme loading at least by a factor of two. The sensitivity of the sensor toward the analyte was slightly increased for the high concentration range, but slightly decreased for the low concentration range: the high noise level (i.e. $43.5 \mathrm{pA}$ ) sets the detection limit of the sensor (calculated as three times the noise level) at $0.28 \mathrm{mg} / \mathrm{ml}$.

\section{Discussion and concluding remarks}

The present rapid response of s-BLMs to peroxide suggests a fast alteration of ion transport through s-BLMs. Lipid membranes composed from phopsphatidylcholine has been proven to be susceptible to dipolar potential alterations which can be triggered due to the enzyme analyte interactions at the membrane surface, forming transient pores in the bilayer structure (Nikolelis and Siontorou [17]). There were no discernable threshold transients observed during our present experiments using s-BLMs suggesting that s-BLMs may differ significantly in structure from planar BLMs. The short time delay (seconds) for peroxide detection when using s-BLMs in comparison to that observed when using planar freely suspended and filter supported BLMs (minutes) is probably due to structure and mechanism of ion current formation associated with the silver metal electrode that supports s-BLMs (Hianik et al. [18]): one side of the s-BLMs (surface of one monolayer) is in contact with the aqueous electrolyte solution; the second monolayer contacts the metal surface, with some regions (depending on the metal roughness) potentially trapping electrolyte solution. The very limited trapped volume of solution makes it possible to reach equilibrium in terms of ion concentration and diffusion very rapidly. In our experiments, the metal-supported BLMs remained stable for periods of over $48 \mathrm{~h}$. The currents through BLMs stabilized within $22 \pm 13$ min on average after immersion of the metal wire with the lipid coating into the electrolyte solution without the analyte. The $22 \pm 13$ min period required before commencement of measurements when 
using wire with a $1.00 \mathrm{~mm}$ diameter is shorter than previously reported times (Siontorou and Nikolelis [19]). This is due to the use of PC alone instead of PC mixtures for the preparation of a lipid solution. The use of dipalmitoylphoshpatidylecholine mixtures also alters the specific capacitance of s-BLMs from that observed when only PC is used. The present minisensor responds to increases of peroxide concentration in solution and can be suitable for monitoring peroxide forming or peroxide catalysed reactions. Whether the latter can be feasible remains to be proved by experimentation: increments of electrolyte should be added so as to lower the peroxide concentration and observe whether there is a recordable decrease of ion current. For practical applications to sensing, a fresh sensing membrane should be prepared for determination of an unknown sample. This ensures elimination of carryover effects (Nikolelis and Siontorou [20]). This also avoids BLM destabilization, which can occur in dry cycling (i.e., transfer of the s-BLM to another electrolyte solution without analyte) due to dehydration of a BLM, or repetitive washing out of peroxide after use. In conclusion, the results indicate that peroxide can be rapidly screened using the present metal supported BLM-based minisensor. The approach provides response times of seconds and detection sensitivity and limits for peroxide that are suitable for direct analysis of industrial or environmental samples without preconcentration. While the work presented here represents an attractive configuration and application of electrochemistry of BLM-based sensors, the practical use of such a sensor for real world applications needs to be further researched to allow commercialization of the present device.

\section{Acknowledgement}

Financial support from the University of Piraeus Research Centre is kindly acknowledged.

\section{References}

[1] Filonov A.E., Duetz W.A., Karpov A.V., Gaiazov R.R., Kosheleva I.A., Competition of plasmid-hearing pseudomonas-putida strains catabolizing naphthalene via various pathways in chemostat culture, Applied Microbiology and Biotechnology, 48, 493-498, 1997

[2] Reshetilov A.N., Iliasov P.V., Filonov A.E., Gayazov R.R., Kosheleva I.A., Boronin A.M., pseudomonas-putida as a receptor element of microbial sensor for naphthalene detection, Process Biochemistry, 32, 487-493, 1997

[3] Van Hamme J.D., Singh A., Ward O.P., Recent advances in petroleum microbiology, Microbiology and Molecular Biology Reviews, 67, 503549, 2003

[4] Paton G.I., Reid B.J., Semple K.T., Application of a luminescence-based biosensor for assessing naphthalene biodegradation in soils from a manufactured gas plant, Environmental Pollution, 157, 1643-1648, 2009

[5] Merks R.M.H., Glazier J.A., A cell-centered approach to developmental biology, Statistical Mechanics and its Applications, 352, 113-130, 2005 
[6] Chattopadhyay K., Mazumdar S., Structural and conformational stability of horseradish peroxidase: effect of temperature and $\mathrm{pH}$, Biochemistry, 2000, 39, 263-270

[7] Veitch N.C., Horseradish peroxidase: a modern view of a classic enzyme, Phytochemistry, 65, 249-259, 2004

[8] Zhang W. \& Li G., Third-generation biosensors based on the direct electron transfer of proteins, Analytical Sciences, 20, 603-609, 2004

[9] Gorton L., Jönsson-Pettersson G., Csöregi E., Johansson K., Dominguez E., Varga G., Amperometric biosensors based on an apparent direct electron transfer between electrodes and immobilized peroxidases, Analyst, 117, 1235-1241, 1992

[10] Ruzgas T., Csöregi E., Emnéus J., Gorton L., Marko-Varga G., Peroxidase-modified electrodes: fundamentals and application, Analytica Chimica Acta, 330, 123-138, 1996

[11] Ghindilis A.L., Atanasov P., Wilkins E., Enzyme-catalyzed direct electron transfer: Fundamentals and analytical applications, Electroanalysis, 9, 661674, 1997

[12] Yang S., Li Y., Jiang X., Chen Z., Lin X., Horseradish peroxidase biosensor based on layer-by-layer technique for the determination of phenolic compounds, Sensors and Actuators B, 114, 774-780, 2006

[13] Azevedo A.M., Martins V.C., Prazeres D.M.F., Vojinović V., Cabral J.M.S., Fonseca L.P., Horseradish peroxidase: a valuable tool in biotechnology, Biotechnology Annual Review, 9, 199-247, 2003

[14] Ottova A.L., Tien H.T., Bilayer lipid membranes: An experimental system for biomolecular electronic devices development, Progress in Surface Science, 41, 337-446, 1992

[15] Tien H.T., Wurster S.H., Ottova A.L., Electrochemistry of supported bilayer lipid membranes: background and techniques for biosensor development, Bioelectrochemistry and Bioenergetics, 42, 77-94, 1997

[16] Dimitrov D.S., Jain R.K., Membrane Stability, Biochimica et Biophysica Acta, 779, 437-468, 1984

[17] Nikolelis D. P, Siontorou C.G., Bilayer-lipid membranes for flowinjection monitoring of acetylcholine, urea, and penicillin, Analytical Chemistry, 67, 936-944, 1995

[18] Hianik T., Passechnik V.I., Sargent D.F., Dlugopolsky J., Sokolikova L., Surface potentials and solvent redistribution may explain the dependence of electrical and mechanical properties of supported lipid bilayers on applied potential and bilayer history, Bioelectrochemistry and Bioenergetics, 37, 61-68, 1995

[19] Siontorou C.G., Nikolelis D.P., A triazine herbicide minisensor based on surface-stabilized bilayer lipid membranes, Analytical Chemistry, 69, 3109-3114, 1997

[20] Nikolelis D.P, Siontorou C.G., Flow injection monitoring and analysis of mixtures of simazine, atrazine, and propazine using filter-supported bilayer lipid membranes (BLMs), Electroanalysis, 8, 907-912, 1996 